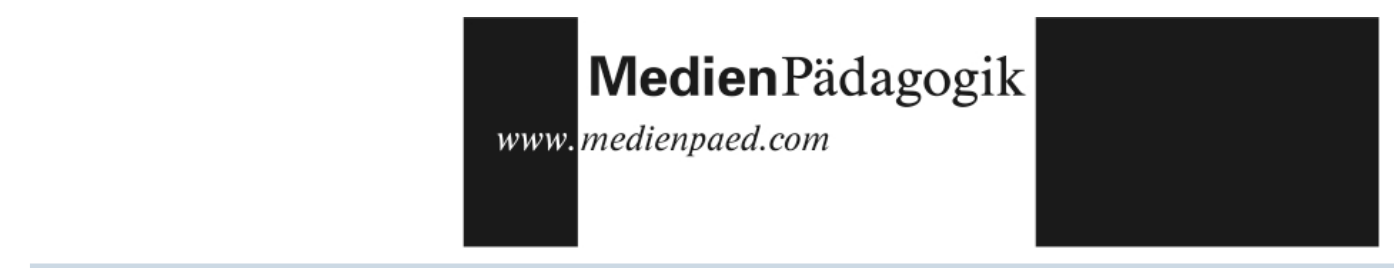

Rezensionen

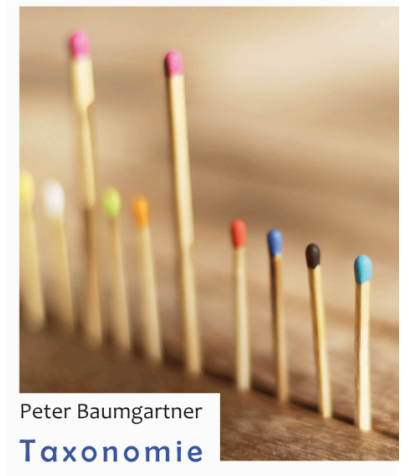

von Unterrichtsmethoden Ein Plädoyer für didaktische Vielfalt
Peter Baumgartner

Taxonomie von Unterrichtsmethoden - Ein Plädoyer für didaktische Vielfalt

Münster, New York, München, Berlin: Waxmann. 276 Seiten, 2011

ISBN: 3-8309-2546-8

$€$ 44,90; CHF 54,20

\section{Ein Orientierungsrahmen für die Vielgestalt didaktisch-methodischen Handelns in der Praxis}

Wie können didaktische Methoden so geordnet und systematisiert werden, dass ihre Zusammenhänge, ihre Stärken, Schwächen und Einsatzbereiche deutlich werden - dass einerseits Lehrende in der Praxis Anregung und Unterstützung für methodische Vielfalt erhalten, andererseits die theoretische Reflexion in der Didaktik angeregt wird? In einer Reihe von Aufsätzen hat sich Peter Baumgartner in der Vergangenheit diesen Fragen gewidmet. Das (vorläufige) Ergebnis seiner Überlegungen liegt nun im Waxmann-Verlag als «Taxonomie von Unterrichtsmethoden» vor, die gleichzeitig als «Ein Plädoyer didaktische Vielfalt» verstanden werden soll.

Auf seinem Weg hat sich Baumgartners Fragestellung von der Wiederverwertbarkeit von Lernobjekten im eLearning (27) erweitert, so dass dass er nun ein allgemeines Gliederungssystem für Unterrichtsmethoden vorstellt (54), in dem auch die Besonderheiten des elektronischen Lehrens und Lernens aufgehoben sind. Damit integriert Baumgartner das Lehren und Lernen mit dem Computer in das allgemeine Feld der Didaktik; eLearning ist für inn kein didaktischer Sonderbereich mit eigenen Gesetzen und theoretischen Bezügen. Stattdessen führt Baumgartner den Werkzeugbegriff in sein didaktisches Modell ein, der es erlaubt, 〈analoge> Methoden für das eLearning zu adaptieren. 


\section{Rezensionen}

Das Buch gliedert sich in drei Teile. In den ersten beiden Teilen ( $« \mathrm{O}$ rientierung und Integration» und «Transfer und Innovation») mit fünf bzw. drei Kapiteln entwirft Baumgartner aufbauend auf den Arbeiten Karl-Heinz Flechsigs ein Orientierungsraster und begründet es. Dabei werden im ersten Teil die theoretischen Grundlagen einer Taxonomie ausgearbeitet und im zweiten wird ihre heuristische Funktion untersucht. Der Schwerpunkt des dritten Teils («Explorationen») mit zwei Kapiteln liegt auf der beispielhaften Anwendung der Taxonomie.

Baumgartners Ausgangspunkt ist die Feststellung, dass ein theoretisch befriedigender Orientierungsrahmen fehlt, mit dem die Vielgestalt didaktisch-methodischen Handelns in der Praxis geordnet und bewertet werden kann. Das habe, so Baumgartner, in der Praxis Methodenarmut zur Folge, da zu wenig über den Zusammenhang zwischen Inhalt und Methode bekannt sei. «Als implizite Folge scheint es beliebig, welche Unterrichtsmethode für welches inhaltliche Ziel verwendet wird» (23). Diese Lücke soll eine didaktische Taxonomie füllen, ein systematisches «Klassifikationsschema von Unterrichtssituationen, die nach einheitlichen sachlogischen pädagogischen Prinzipien, Verfahren und Regeln beschrieben werden können» (22).

Baumgartner arbeitet im dritten und vierten Kapitel zwei Dimensionen der didaktischen Beschreibung von Unterrichtswirklichkeit heraus. Zum einen grenzt er «Handlungsbereiche» voneinander ab, die sich in der Reichweite der didaktischen Gestaltung unterscheiden (Abb. 3.3 und 3.8):

- Didaktische Interaktionen als Handlungseinheiten von wenigen Sekunden bis Minuten

- Didaktische Szenarien als didaktische Einheiten von mehreren Minuten bis zu einer Stunde

- Didaktische Ensembles als inhaltliche Einheiten von mehreren Stunden

- Module als curriculare Einheiten von 1 bis 30 ECTS

- Curricula als Ausbildungseinheiten, z.B. ganzer Studiengänge von 120 bzw. 180 ECTS 


\section{Rezensionen}

Diese Handlungsbereiche lassen sich als Ebenen einer inklusiven Hierarchie auffassen, bei der die höheren Stufen die jeweils darunterliegenden umfassen. Auf jeder Ebene steht ein anderer Bereich mit spezifischen Zusammenhängen im Fokus.

Die zweite Dimension bilden die «didaktischen Beschreibungsstufen», mit denen der Abstraktionsgrad der Beschreibung von Unterricht charakterisiert wird. Baumgartner führt sechs Beschreibungsstufen an (Abb. 4.19), die er genauer ausführt:

- Praxisbeschreibungen

- Muster

- Modelle

- Prinzipien

- Dimensionen

- Kategorien

Muster und Modelle sind zwei Abstraktionsstufen von Methodenbeschreibungen und bilden den Kern von Baumgartners Konzeption. Dabei liegt inm besonders am Herzen, «die Nützlichkeit dieser Stufe der Musterbeschreibungen im Rahmen eines ganzheitlichen pädagogisch-didaktischen Gesamtkonzeptes (...) deutlich zu machen» (74). Aus der Kombination dieser beiden Dimensionen ergibt sich im fünften Kapitel der Orientierungsrahmen der didaktischen Taxonomie, dessen Zeilen und Spalten bzw. deren Schnittpunkte jeweils unterschiedliche Abstraktionsgrade didaktischer Beschreibungen unterschiedlicher Reichweite beinhalten. Mit diesem Instrumentarium können auch die Begriffe Didaktisches Design, Didaktisches Arrangement, Handlungsprozesse, Handlungsstrukturen Verlaufsformen des Unterrichts näher bestimmt werden.

Im zweiten Teil des Buches vertieft Baumgartner seine Untersuchung zu den 〈inneren' Beschreibungsstufen 〈Muster〉, 〈Modell $\rangle,\langle$ Prinzipien〉 und 〈Dimensionen〉. Am Beispiel der 〈KugellagerMethode> beschreibt er eine Unterrichtsmethode als Muster und als Modell. Die didaktischen Prinzipien und Dimensionen stellen dagegen den Zusammenhang zwischen den Methoden und Baumgartners didaktischem Kategorialmodell her. 


\section{Rezensionen}

Nach einer theoretischen Beschreibung seiner Dimensionalen Analyse in Kapitel sieben entwickelt Baumgartner ein Set von 26 Didaktischen Dimensionen mit insgesamt 130 Didaktischen Prinzipien, die er aber als noch nicht abgeschlossen verstanden sehen möchte. Sie können zum einen noch erweitert werden, zum anderen könnten noch treffendere Bezeichnungen für die einzelnen Dimensionen gefunden werden (212). Zur Veranschaulichung dieses recht komplexen Systems erstellt Baumgartner damit für die bereits bekannte ,Kugellager-Methode' ein didaktisches Profil.

Im dritten Teil wendet Baumgartner seine Überlegungen - nach einer Analyse des Beschreibungssystems von Flechsig - im bei weitem umfangreichsten Kapitel auf die von Flechsig vorgestellten Modelle an. So kann er die dort vorgefundenen Beschreibungen präzisieren. Ziel ist es «zu zeigen, wie eine Nutzung der Taxonomie als kognitives Werkzeug in ihrer heuristischen Funktion aussieht und welche Ergebnisse dabei erwartet werden können» (221).

Damit legt Baumgartner ein anspruchsvolles Gedankengebäude vor, das das Potenzial zu einem didaktischen Klassiker hat. Der Gestaltungsmusteransatz als noch relativ junge Bewegung in der Didaktik erhält hier eine mögliche theoretische Rahmung, die inn zu den älteren Theorielinien der Allgemeinen Didaktik anschlussfähig macht Baumgartner stellt sich hier ausdrücklich in die Tradition der geisteswissenschaftlich orientierten Didaktik-Diskussion (24). Er entwickelt einen systematischen Ordnungsrahmen für Unterrichtsmethoden, der in zukünftigen Forschungen mit Inhalten gefüllt werden kann und muss.

Nicht voll eingelöst wird dagegen genau genommen das Versprechen aus dem Untertitel nach didaktischer Vielfalt, da inhaltsdidaktische Fragen - die Fragen danach, was aus welchen Gründen gelehrt werden soll - nicht weiter ausdifferenziert werden.

An vielen Stellen diskutiert Baumgartner grundlegende Konzepte und Begriffe, wie etwa den Gegenstandsbereich der Didaktik oder verschiedene Methodenverständnisse, erörtert ihre Inhalte und Grenzen. Damit eignet es sich sehr gut um einen Überblick über die Grundfra- 


\section{Rezensionen}

gen, -probleme und -begriffe der Didaktik zu erhalten. Allerdings werden Begriffe teilweise abweichend vom 〈Mainstream〉 gefasst und auch der sehr strukturierte (aber durchaus nicht humorlose) Schreibstil Baumgartners erschwert an manchen Stellen die Lektüre.

Mit diesem Entwurf möchte Baumgartner die Diskussion in der Didaktik anstoßen. Dafür verweist er auch auf eine begleitende Webseite zu dem Buch, auf der nicht nur Errata und Zusatzmaterialien zu finden sind sondern die auch zur Weiterentwicklung der Idee einladen soll. Dort charakterisiert er sein Werk, eine Äußerung Flechsigs aufnehmend, als Buch für Kartografen und noch nicht als Karte für den Wanderer.

Stephen Frank 